\title{
Preface for the special feature
}

\author{
Stefano Marmi ${ }^{1}$
}

Published online: 13 June 2015

(C) Japan Association for Evolutionary Economics 2015

Why? Why one more special issue on mathematical methods in economics? What has yet to be said on the subject beyond what has already been said? Is there some true meat to add or is it just one more academic exercise (which may well be worth doing but perhaps of limited scope)?

These are some of the questions that came to my mind when I was asked to write a preface to this collection of papers. As a mathematician with a keen albeit amateurish interest in economics and finance I feel deeply the need of more interaction between mathematics and these two disciplines. Thus in writing this preface I have a natural positive bias.

Traditionally the goal of this kind of special issue is to help to bring together those mathematicians who are seriously interested in obtaining new challenging stimuli from economic theories and those economists who are seeking effective mathematical tools for their research. I think however there is a little bit more to be said today.

Mathematics is perhaps the cheapest of natural sciences (this is at least the point of view of Vladimir Arnold). It can also be seen as a language. Historically this language has been proven to be very useful for understanding nature. The degree of success that mathematics has had in helping social sciences is more controversial. Economics is a social science that sometimes suffers of a strange sort of illness, "physics envy". According to Andrew Lo, "physics envy" is the "desire to be able to explain $99 \%$ of all economic phenomena with three laws. That's what physicists can do [in physics]. In fact, [in economics] we have 99 laws that explain maybe $3 \%$ of all phenomena".

Stefano Marmi

s.marmi@sns.it

1 Scuola Normale Superiore di Pisa, Pisa, Italy 
I think Lo's warning is correct and I agree with the point he makes that "physics envy" is widespread in contemporary economics.

It should not be so. Economics often deals with situations that have significantly more uncertainty than those physics addresses. Moreover we should not forget that human behaviour is not nearly as stable and predictable as physical phenomena. To complicate the task even further the knowledge which is developed in economics and in finance can often be immediately applied and this sometimes affects the behaviour of agents to such an extent that any discovery should be an object of constant scrutiny to be sure that its "truth" persists in time. A worry that mathematicians do not have. A theorem is forever (...better than diamonds).

For all the above reasons I am convinced that there is a lot to do in developing new mathematical methods in economics. This special issue is a fine example of what should and can be done in this direction. It focuses on a rather underexplored direction, namely, the use of geometric tools such as hyperplane arrangements, tropical geometry, polytopes, and others to model new and old problems in economics, like the theory of collective choice, of demand, production theory, etc.

In the first part of the special issue the problem of social choice is discussed with a contribution by Donald Saari and another by Amendola, Marengo, Pirino, Settepanella, and Takemura.

Saari tries to go beyond the reductionist point of view in developing a system's analysis challenge to social choice that instead of "cutting the links that connect parts" tries to "determine how to discover appropriate connecting links". The reward is substantial, since "once these links are understood, resolutions can be crafted".

Amendola and co-authors look at choice over a multidimensional space of possibilities. Their work combines algebraic (graphs), geometric (hyperplanes arrangements), numerical (combinatorial), and probabilistic approaches in a general framework in order to make problems more tractable.

The second part of the special issue contains three contributions to a rather new field, tropical geometry in economics. Tropical geometry is a relatively young mathematical discipline, which has been growing very rapidly during the last 15 years. It is based on simple (piecewise linear) constructions that have had strong applications to enumerative algebraic geometry. An introduction to this topic is provided by and it is followed by, respectively, its application to international trade by Shiozawa and one on Perron rankings by Ngoc.

The final article of the special issue by Dosi, Grazzi, Marengo, Ponchio, and Settepanella develops a purely geometric framework to assess firm level heterogeneity and to study the rate and direction of technical change. Building on previous work by Hildenbrand they use the geometry of the zonotope composed by vectorsfirms in a narrowly defined industry (its volume and its orientation in space) to investigate inter-firm heterogeneity and to measure the rates and directions of technical change over time. This is the part of the special issue I am most familiar with since I have been lucky to discuss it several times with the authors. I am convinced that their intuition is sound and that there is much more to be done in this direction. One thing to try is probably to combine their approach with a dynamical point of view.

Enjoy the reading. 